Victorino Farga $C$.

\title{
Formularios de baciloscopía: ¿son un instrumento adecuado para el diagnóstico oportuno y seguimiento de la tuberculosis?
}

\author{
PAULINA RAMONDA C.*, PAULINA PINO Z.** y PATRICIA CANTILLANA R.***
}

Smear forms: an appropriate instrument for early diagnosis and monitoring of tuberculosis?

Background: Smear forms (BK) should provide some crucial patient's information that permits the laboratory to select the appropriate bacteriological techniques for each case, a process that would fail if such information was inaccurate or omitted. Objective: To evaluate the quality of information contained in BK forms sent to laboratory for individual's diagnosis or treatment monitoring of tuberculosis (TB) in the Chilean Health Services. Methods: Descriptive study based on secondary data of all forms of smear-positive $(B K+)$ in $2012(n=232)$ obtained from the population allocated to the South Metropolitan Health Service. BK+ forms were matched to BK+ cases handled in the TB program to assess the validity or omission of information on treatment situation (previous or current) and the current follow-up time. Results: Diagnostic BK were $62.9 \%$ (16.4\% with previous treatment); of these $74.7 \%$ correctly reported a history of TB treatment but in patients previously treated the percentage falls to $66.7 \%$. Among those under treatment control $26.7 \%$ were classified as previously treated and $43 \%$ did not recorded the follow-up time. Discussion: BK forms omit or incorrectly recorded fundamental information that should lead the laboratory procedures. As the finding of TB involves each and every one in the health personnel, we recommend continuous training stressing the inclusion of treatment's history and risk factors while the forms should avoid ambiguity.

Key words: Tuberculosis, pulmonary tuberculosis, smear forms.

\section{Resumen}

Introducción: El formulario de baciloscopía (BK) debe aportar información esencial para que el laboratorio seleccione las técnicas bacteriológicas correspondientes, las cuales dejan de realizarse si ésta es incorrecta u omitida. Objetivo: Evaluar la calidad de la información contenida en los formularios de BK enviados al laboratorio para diagnóstico o control de tratamiento de la tuberculosis (TB) en los servicios de salud en Chile. Métodos: Estudio descriptivo con datos secundarios, de todos los formularios de BK con resultado positivo $(B K+)$ en $2012(n=232)$, obtenidos de la población asignada al Servicio de Salud Metropolitano Sur. Los formularios de BK+ del laboratorio se cotejaron con los casos $B K+$ manejados en el programa-TB, para evaluar la validez u omisión de la situación de tratamiento (previo o actual) y el tiempo de tratamiento. Resultados: Las BK+ de diagnóstico fueron $62,9 \%$ (16,4\% con tratamiento anterior); 74,7\% reporta correctamente el antecedente de tratamiento antituberculoso, pero en los pacientes tratados anteriormente esa proporción baja a $66,7 \%$. De las $B K+$ de control de tratamiento $(37,1 \%)$, el $26,7 \%$ se catalogan como pacientes antes tratados y el 43\% no registra el tiempo que está bajo tratamiento. Discusión: En los formularios de BK se omite o registra erróneamente información clave para el proceder del laboratorio. Puesto que la pesquisa de casos involucra a todo el personal de salud, se recomienda un entrenamiento constante que preconice la inclusión de antecedentes de tratamiento y de factores de riesgo en los formularios, al tiempo que estos deben evitar ambigüedades.

Palabras clave: Tuberculosis, tuberculosis pulmonar, formulario de baciloscopía.

\footnotetext{
* Médico Neumotisióloga. Servicio de Salud Metropolitano Sur.

** Profesora asociada, Escuela de Salud Pública. Facultad de Medicina, Universidad de Chile.

***Bioquímica encargada de las actividades del Laboratorio de Referencia de Tuberculosis del Servicio de Salud Metropolitano.
} 


\section{Introducción}

La microbiología es el pilar fundamental del diagnóstico de la tuberculosis (TB), aislando al bacilo y demostrando la presencia del Mycobacterium tuberculosis en alguna muestra orgánica ${ }^{1}$. Las técnicas bacteriológicas que pesquisan los casos incluyen la baciloscopía (BK) y el cultivo². El rendimiento de dichas técnicas depende de la localización de las lesiones, de la cuantía de la población bacilar y de la calidad de la muestra ${ }^{1,2}$. A su vez la localización de casos es una actividad de salud pública, que a través de tamizajes sucesivos de los sintomáticos respiratorios (SR) es capaz de pesquisar precozmente a los pacientes con tuberculosis pulmonar bacilífera, que constituyen la fuente de transmisión de la enferme$\mathrm{dad}^{2,3}$. Cuando se requiere identificar pacientes con escasa cantidad de bacilos (TB de los niños, lesiones no abiertas a bronquios o localizaciones extrapulmonares), con posible resistencia a drogas antituberculosas o con micobacterias no tuberculosas, el laboratorio efectúa cultivo de Koch -en laboratorios de referencia asignados a los servicios de salud-, estudios de susceptibilidad a drogas y la tipificación de micobacterias -que son derivadas al Instituto de Salud Pública de Chile (ISP) - 2,4,5 (Tabla 1).

La solicitud de investigación bacteriológica de tuberculosis se realiza a través de un formulario (Anexo), basado en el de la Organización Mundial de la Salud (OMS), Departamento "Alto a la tuberculosis" ". En Chile el protocolo incluye dos BK de pesquisa cuando un paciente consulta con síntomas respiratorios y se cultiva al menos una de las muestras. Para el control de tratamiento (CT) se solicita una BK mensual de expectoración (en las TB pulmonares) y tres BK de orina (en las TB génito-urinarias), agregando cultivo si la BK de CT del $4^{\circ}$ mes en adelante se encuentra positiva (por la posibilidad de confirmar fracaso de tratamiento) y estudio de susceptibilidad a drogas en caso de que dicho cultivo resulte positivo ${ }^{2,4}$.

El formulario de solicitud de baciloscopía representa la primera instancia en el aporte de los antecedentes de los pacientes, para que el laboratorio especializado seleccione las técnicas bacteriológicas que corresponde ejecutar específicamente en cada situación clínica ${ }^{2,6}$, las cuales dejarán de realizarse si los datos no son informados correctamente. Pese a que el formulario está incluido en las Normas Técnicas del Programa

Tabla 1. Técnicas de laboratorio que se realizan según antecedentes para el tamizaje y control de tratamiento de la tuberculosis en Chile

\begin{tabular}{|c|c|}
\hline Antecedentes & Técnicas de laboratorio que se realizan \\
\hline Pesquisa & $\begin{array}{l}2 \text { BK expectoración en SR y } 1 \text { cultivo } \\
6 \text { BK orina o } 1 \text { BK de otros líquidos } \\
\text { con } 1 \text { cultivo por cada muestra en TBC extrapulmonar }\end{array}$ \\
\hline Control mensual de tratamiento & $\begin{array}{l}1 \text { BK expectoración } \\
3 \text { BK orina en TB renal }\end{array}$ \\
\hline $\begin{array}{l}\text { Posible fracaso de tratamiento } \\
\left(\mathrm{BK}(+) \text { de } \mathrm{CT} 4^{\circ} \text { mes en adelante) }\right. \\
\text { Cultivo }(+) \text { de } \mathrm{CT} 4^{\circ} \text { mes en adelante }\end{array}$ & $\begin{array}{l}\text { Cultivo } \\
\text { Estudio de susceptibilidad }\end{array}$ \\
\hline $\begin{array}{l}\text { TB con probable escasa población bacilar } \\
\text { Niños }<15 \text { años, TB EP, contactos TB }\end{array}$ & BK y cultivo de cada muestra \\
\hline Población cerrada: Hogares, cárceles... & BK y cultivo de cada muestra \\
\hline Antecedentes de tratamiento (AT) recaídas, abandonos & BK, cultivo, estudio susceptibilidad \\
\hline Personal de salud & BK, cultivo, estudio susceptibilidad \\
\hline Inmigrantes & BK, cultivo, estudio susceptibilidad \\
\hline Contacto de MDR & BK, cultivo, estudio susceptibilidad \\
\hline PVVIH/SIDA (Co-infección retroviral) & $\begin{array}{l}\text { BK, cultivo, estudio susceptibilidad y tipificación de } \\
\text { micobacterias }\end{array}$ \\
\hline $\begin{array}{l}\text { Cultivo }(+) \text { de orina, poli-resistencia a drogas } \\
\text { antituberculosas, cultivo }(+) \text { de ganglio } \\
<15 \text { años o cultivo }(+) \text { tejido óseo en }<15 \text { años }\end{array}$ & Tipificación de micobacterias \\
\hline
\end{tabular}


Nacional de Control de la Tuberculosis ${ }^{2}$ la solicitud, en el Servicio de Salud Metropolitano Sur (SSMS) se hace con diversos instrumentos (ocho tipos), que se resumen en la Tabla 2. Los formularios que se asemejan a lo normado incluyen la clasificación del paciente según haya recibido tratamiento antituberculoso -virgen a tratamiento (VT) o antes tratado (AT)-y además incluye espacios específicos para el registro de las drogas recibidas, en episodios anteriores para los AT y en el episodio actual si la BK es de control de tratamiento.

La incidencia de tuberculosis en Chile en el último quinquenio se ha mantenido estacionaria (cercana a 13/100.000) y considerando la nueva meta sanitaria para el decenio (tasa morbilidad de $5 / 100.000)^{7}$ se debe optimizar la gestión del diagnóstico y tratamiento de la TB. La adecuada coordinación entre el ámbito clínico y el laboratorio es imprescindible para el éxito de este manejo.

El propósito de este estudio fue evaluar la calidad de la información contenida en los formularios de BK enviados al laboratorio para diagnóstico o control de tratamiento de la TB en los servicios de salud en Chile. Se trató de conocer si el llenado de la solicitud de BK se realiza de acuerdo a la norma y si las muestras enviadas son de buena calidad; cuantificando si la información está completa, si es veraz y, eventualmente, registrando las fallas de información que pueden limitar o retrasar el proceder del laboratorio. Esta información podrá orientar futuras intervenciones para el diagnóstico más oportuno y el buen seguimiento del tratamiento de los pacientes.

\section{Métodos}

Se realizó un estudio descriptivo con datos secundarios, que incluyó a todos los formularios de solicitud de BK de 2012 con resultado positivo
$(\mathrm{BK}+)$ del laboratorio de referencia del Servicio de Salud Metropolitano Sur (SSMS). Estos fueron obtenidos de la población asignada al SSMS correspondiente a 1.053.418 habitantes (proyección Censo de 2002), distribuidos en 11 comunas con un total de 35 Consultorios y Centros de Salud Familiar ${ }^{8}$. El laboratorio de referencia procesa las muestras de pacientes provenientes de la red asistencial, integrando a otros cinco laboratorios. El conjunto de formularios estudiados incluye a los de pesquisa y de control de tratamiento, de muestras pulmonares y extra-pulmonares, procedentes de la atención primaria de salud (APS), hospitales, centro de diagnóstico y tratamiento (CDT) y servicios de urgencia.

La información contenida en los formularios -demográfica, factores de riesgo para desarrollar tuberculosis y asociación con otras enfermedades - se cotejó con la información registrada para los mismos casos $\mathrm{BK}+$ en la base de datos del Programa TB de 2012. Esta incluye a todos los pacientes con tuberculosis de localizaciones pulmonares o extra-pulmonares (EP), niños y adultos, sin tratamientos previos antituberculosos (VT) y antes tratados (AT) -AT por recaídas y AT por abandonos (también denominados AT después de pérdida de seguimiento $)^{6}$. Asimismo en este estudio se incluyó los formularios que demostraron micobacterias no tuberculosas, los de personas viviendo con el virus de la inmunodeficiencia humana (PVVIH), de los que fallecieron con $\mathrm{BK}+\mathrm{y}$ de los residentes de otros Servicios de Salud si sus muestras resultaron positivas en el Laboratorio de Referencia del SSMS. Conjuntamente, en los formularios de diagnóstico de TB de las muestras de expectoración se evaluó la calidad y se relacionó con el grado de positividad. No hubo pérdidas de información ni exclusión de formularios, conformándose un total de 232 formularios con resultado positivo.

Tabla 2. Tipos de formularios para investigación bacteriológica de tuberculosis utilizados en el Servicio de Salud Metropolitano Sur en 2012

\begin{tabular}{|lccccc|}
\hline $\begin{array}{l}\text { Tipo de } \\
\text { formulario }\end{array}$ & $\begin{array}{c}\text { Especifica } \\
\text { VT/AT } \mathbf{y} \\
\text { drogas AT/CT }\end{array}$ & $\begin{array}{c}\text { Cantidad de tipos } \\
\text { de formularios } \\
(\mathbf{n})\end{array}$ & $\begin{array}{c}\text { BK+ diagnóstica } \\
(\mathbf{n}=\mathbf{1 4 6}) \\
\mathbf{n}(\mathbf{\%})\end{array}$ & $\begin{array}{c}\text { BK+ control } \\
\text { tratamiento }(\mathbf{n}=\mathbf{8 6}) \\
\mathbf{n}(\mathbf{\%})\end{array}$ & $\begin{array}{c}\text { BK+ total } \\
(\mathbf{n}=\mathbf{2 3 2}) \\
\mathbf{n}(\%)\end{array}$ \\
$\begin{array}{l}\text { Adecuado a } \\
\text { norma }\end{array}$ & Ambas & 4 & $69(47,3)$ & $35(40,7)$ & $104(44,8)$ \\
$\begin{array}{l}\text { Parcialmente } \\
\text { adecuado a norma }\end{array}$ & Sólo VT/AT & 2 & $72(49,3)$ & $51(59,3)$ & $123(53,0)$ \\
$\begin{array}{l}\text { No adecuado } \\
\text { a norma }\end{array}$ & Ninguna & 2 & $5(3,4)$ & $0 \quad(0,0)$ & $5(2,2)$ \\
\hline
\end{tabular}

$\mathrm{BK}+=$ baciloscopía con resultado positivo; $\mathrm{VT} / \mathrm{AT}=$ virgen a tratamiento/antes tratado; $\mathrm{AT} / \mathrm{CT}=$ antes tratado/control de tratamiento. 
El análisis se orientó a la estimación de la prevalencia de omisiones y de errores en los formularios de $\mathrm{BK}+$, en comparaciones entre el nivel primario de atención y niveles de mayor complejidad, entre los tipos de formularios y entre la calidad de las muestras (clasificada por los laboratorios), registrada específicamente en los formularios de expectoración. Para tales análisis se utilizó el Programa STATA®, versión $11.0^{9}$.

El protocolo fue revisado y aprobado por el Comité Ético de Investigación Clínica del SSMS en octubre de 2013. Los identificadores individuales fueron omitidos de los archivos, de forma de resguardar la confidencialidad.

\section{Resultados}

Se contó con 232 formularios del laboratorio de referencia del SSMS con diversos grados de positividad (de escasos 4-9 bacilos ácido-alcohol resistentes $(\mathrm{BAAR}),+1,+2$, hasta +3 ) pertenecientes a 112 pacientes de 15 o más años con baciloscopía positiva. En escasa proporción se identificó alguna micobacteria no tuberculosa $(7,8 \%)$. Las baciloscopías de diagnóstico fueron

Tabla 3. Procedencia de las muestras de baciloscopía positivas

\begin{tabular}{|lrrr|}
\hline Procedencia & $\begin{array}{c}\text { Diagnóstica } \\
\text { n (\%) }\end{array}$ & $\begin{array}{c}\text { CT } \\
\text { n (\%) }\end{array}$ & \multicolumn{1}{c|}{$\begin{array}{c}\text { Total } \\
\text { n (\%) }\end{array}$} \\
APS & $81(55,5)$ & $69(80,2)$ & $150(64,7)$ \\
Hospitales & $45(30,8)$ & $14(16,3)$ & $59(25,4)$ \\
CDT & $9(6,2)$ & $1(1,2)$ & $10(4,3)$ \\
Urgencia y SAPU & $11(7,5)$ & $2(2,3)$ & $13(5,6)$ \\
Total & $146(62,9)$ & $86(37,1)$ & $232(100,0)$ \\
\hline
\end{tabular}

$\mathrm{CT}=$ control de tratamiento; APS $=$ atención primaria de salud; CDT = centro de diagnóstico y tratamiento; $\mathrm{SAPU}=$ servicios de atención primaria de urgencia.
$62,9 \%$ y las de control de tratamiento $37,1 \%$. La mayor parte de los formularios proceden de la APS $(64,7 \%)-55,5 \%$ de las BK+ de diagnóstico y $80,2 \%$ de las $\mathrm{BK}+$ de $\mathrm{CT}-\mathrm{y}$ el $25,4 \%$ proviene de hospitales (Tabla 3). Del grupo de formularios $\mathrm{BK}+$ de diagnóstico y de expectoración el 77,0\% especifica la calidad de la muestra, siendo de mala calidad la que corresponde a saliva y en la que predomina como es de esperar, la escasa positividad ${ }^{10}$ con 4-9 BAAR $(\mathrm{p}=0,016)$ (Tabla 4).

Cerca del $10 \%$ de las BK de diagnóstico no señalan procedencia, edad ni domicilio, (realizando la atención primaria de salud mejores registros) y si los formularios no proceden de la APS el $80 \%$ no informa el consultorio en que el paciente deberá ser tratado. El 6,9\% no señala factores de riesgo que están presentes -co-infección con $\mathrm{VIH}$, contacto TB o privación de libertad- lo que determinaría implementar otras técnicas de laboratorio diferentes de la baciloscopía (Tablas 1 y 5$)$.

El 74,7\% reporta correctamente la condición de virgen a tratamiento (VT) o antes tratado (AT). No completa VT/AT $13,7 \%$ o lo describe erróneamente $11,6 \%$. Destaca que esto es diferente si se trata de pacientes tratados anteriormente, ya sea por recaídas o abandonos recuperados. En los formularios de baciloscopía de diagnóstico $(16,4 \%)$ la proporción en que el dato AT se señala correctamente baja a $66,7 \%$. No se describen las drogas recibidas en $91,7 \%$ de los AT, incluso en $63,6 \%$ de los formularios que tienen efectivamente como aportar el dato (Tabla 5).

En la Tabla 6 se presenta la situación de las BK de control de tratamiento (CT) con resultado positivo. El $24,4 \%$ no especifica que se trata de esa condición (CT) y el 43\% no señala el número de meses que está recibiendo tratamiento antituberculoso, no produciendo la alerta en el laboratorio ante un posible fracaso. El 26,7\% de los solicitantes de baciloscopías confunden la terminología del formulario, declarando Antes

Tabla 4. Grado de positividad* de la baciloscopía de diagnóstico de expectoración según calidad de la muestra

\begin{tabular}{|c|c|c|c|c|}
\hline \multirow[t]{2}{*}{ Grado de positividad } & \multicolumn{2}{|c|}{ Calidad de la muestra } & \multirow{2}{*}{$\begin{array}{c}\text { Total } \\
\text { n }(\%) \\
107(100,0)\end{array}$} & \multirow[t]{2}{*}{$\mathbf{p}^{* *}$} \\
\hline & $\begin{array}{c}\text { Buena } \\
\text { n }(\%) \\
93(86,9)\end{array}$ & $\begin{array}{c}\text { Mala (saliva) } \\
\text { n (\%) } \\
14(13,1)\end{array}$ & & \\
\hline 4-9 BAAR/100 campos & $4(4,3)$ & $3(21,4)$ & $7(6,5)$ & 0,016 \\
\hline+ & $37(39,8)$ & $8(57,1)$ & $45(42,1)$ & 0,220 \\
\hline++ & $26(28,0)$ & $2(14,3)$ & $28(26,2)$ & 0,278 \\
\hline+++ & $26(28,0)$ & $1(7,1)$ & $27(25,2)$ & 0,095 \\
\hline
\end{tabular}

*Número de bacilos ácido alcohol resistentes (BAAR) o número de cruces. *** $\chi^{2}$. 
Tabla 5. Características de los registros de los formularios de baciloscopía de diagnóstico, en el Servicio de Salud Metropolitano Sur en 2012

\begin{tabular}{|c|c|c|}
\hline Formulario de baciloscopía & $\begin{array}{r}\text { BK+ de } \\
\text { (n } \\
\text { n }\end{array}$ & $\begin{array}{l}\text { diagnóstico } \\
=146) \\
(\%)\end{array}$ \\
\hline Sin procedencia & 16 & $(11,0)$ \\
\hline Sin RUN & 2 & $(1,4)$ \\
\hline Sin edad & 14 & $(9,6)$ \\
\hline Sin domicilio & 19 & $(13,0)^{*}$ \\
\hline Sin comuna & 23 & $(15,8)^{*}$ \\
\hline Sin consultorio & 63 & $(43,2)^{*}$ \\
\hline Sin factor de riesgo & 10 & $(6,9)$ \\
\hline Virgen a tratamiento & 122 & $(86,3)$ \\
\hline Registra VT correctamente & 93 & $(76,2)$ \\
\hline Registra VT/AT correctamente & 109 & $(74,7)$ \\
\hline VT/AT: sin dato o errado & 37 & $(25,3)$ \\
\hline No registra VT/AT & 20 & $(13,7)$ \\
\hline VT/AT incorrecto & 17 & $(11,6)$ \\
\hline Antes tratados & 24 & $(16,4)$ \\
\hline Registra AT correctamente & 16 & $(66,7)$ \\
\hline No registra drogas recibidas en $\mathrm{AT}$ & 22 & $(91,7)$ \\
\hline
\end{tabular}

$\mathrm{BK}+=$ baciloscopía con resultado positivo; $\mathrm{RUN}=$ rol único nacional; $\mathrm{VT}=$ virgen a tratamiento, nunca antes tratado con drogas antituberculosas; VT/AT = virgen a tratamiento/antes tratado de tuberculosis; AT $=$ antes tratado. ${ }^{*}$ Diferencia significativa entre el nivel primario de atención y niveles de mayor complejidad $\left(\chi^{2}, \mathrm{p}<0,001\right)$.
Tratado en situaciones en que se trata de Control de Tratamiento. El 55,8\% no reporta las drogas que el paciente recibe en el episodio actual, pero hay clara diferencia entre los formularios con y sin especificación de drogas $(10,4 \%$ vs $89,6 \%$; $<<0,001)$.

\section{Discusión}

En este estudio de formularios de solicitud de investigación bacteriológica del SSMS la frecuencia de micobacterias no tuberculosas $(7,8 \%)$ es semejante a lo aislado en el Instituto de Salud Pública de Chile $(8,7 \%)^{5}$. Pese a que en los formularios de diagnóstico y de expectoración sólo 77\% especifica la calidad de la muestra, el 13,1\% de las BK+ fueron de mala calidad (saliva) y predomina en este grupo la escasa cantidad de BAAR. Esta situación representa una mayor dificultad para el laboratorio e incluso plantea la posibilidad de retraso en el diagnóstico de la TB pulmonar. Si localmente no se supervisa la toma y recepción del examen, las muestras enviadas podrían no proceder del sitio de la lesión. En los formularios de diagnóstico se omite información orientadora para el laboratorio -el $25,3 \%$ no reporta o señala erróneamente el antecedente de tratamiento antituberculoso $\mathrm{y}$ el $6,9 \%$ no reporta factores de riesgo- $\mathrm{y}$ en los de control de tratamiento el $26,7 \%$ falla

Tabla 6. Procedencia y deficiencias de las solicitudes de baciloscopía de control de tratamiento

\begin{tabular}{|lrr|}
\hline Formularios de control de tratamiento $(\mathbf{C T})$ & BK+ Control de tratamiento $(\mathbf{n}=\mathbf{8 6})$ \\
$\mathbf{n}$ & $\mathbf{n}(\mathbf{\%})$ \\
Procedencia & & \\
$\quad$ APS & 69 & $(80,2)$ \\
$\quad$ Hospitales & 14 & $(16,3)$ \\
$\quad$ CDT & 1 & $(1,2)$ \\
$\quad$ Urgencia-SAPU & 1 & $(2,3)$ \\
No registra que es BK de control de tratamiento & 21 & $(24,4)$ \\
Solicita 2 BK de CT en misma fecha & 16 & $(18,6)$ \\
No registra mes de control de tratamiento & 37 & $(43,0)$ \\
No registra que es CT de primer episodio & 34 & $(39,5)$ \\
Confunde CT con AT & 23 & $(26,7)$ \\
No registra drogas en uso durante el CT & 48 & $(55,8)^{*}$ \\
$\quad$ En formularios que especifican drogas en uso & 6 & $(12,5)$ \\
$\quad$ En formularios que no especifican drogas en uso & 42 & $(87,5)$ \\
\hline
\end{tabular}

$\mathrm{CT}=$ control de tratamiento; $\mathrm{BK}+=$ baciloscopía con resultado positivo; $\mathrm{APS}=$ atención primaria de salud; CDT = centro de diagnóstico y tratamiento; $\mathrm{SAPU}=$ servicios de atención primaria de urgencia; $\mathrm{BK}=$ baciloscopía; $\mathrm{AT}=$ antes tratado con drogas antituberculosas. ${ }^{*}$ Diferencia significativa entre tipos de formularios que especifican las drogas antituberculosas comparado con los que no la especifican $\left(\chi^{2}, \mathrm{p}<0,001\right)$. 
al describir las BK de control de tratamiento como pacientes tratados anteriormente. Hemos examinado la situación de estos registros en una población usuaria de un Servicio de Salud de la Región Metropolitana, en un momento que Chile presenta baja prevalencia de tuberculosis, pero con un enlentecimiento en la reducción de la incidencia $^{11}$. Esto supone que la red de laboratorios de TB responda de manera organizada, dando cobertura a los niveles intermedios y locales. No conocemos reportes previos, por lo que cabe preguntarse si esta situación es propia del SSMS o refleja un problema compartido con los demás servicios del país. En el Laboratorio de referencia del Servicio de Salud Metropolitano Oriente el $21 \%$ de los formularios recibidos en el $2^{\circ}$ trimestre de 2011 no cuentan con el antecedente de tratamiento (VT/AT) (Bioquímica Andrea Searle, comunicación personal).

La localización de casos constituye una actividad de salud pública. Para disminuir la transmisión de la tuberculosis todo el personal de salud, cuando se enfrenta a un paciente que consulta con síntomas respiratorios debe involucrarse en la solicitud de la baciloscopía. El tamizaje de ningún modo es una acción específica médica ni exclusiva del equipo técnico de $\mathrm{TB}^{2}$. Hay que considerar que en Chile en 2012 (evaluación del Programa TB del ISP) se realizaron 281.161 baciloscopías y 181.941 cultivos de diagnóstico. Esto permitió pesquisar 1.392 casos de tuberculosis bacilífera y 511 TB sólo por cultivo, pese a que operacionalmente la actividad de pesquisa en sintomáticos respiratorios está muy deteriorada (muy por debajo de la meta de realizar 50 BK de diagnóstico por cada 1.000 consultas de morbilidad de adultos $)^{2,11}$. Se puede postular que además de las deficiencias en la oportunidad de solicitar el examen de BK, de infraestructura de los laboratorios con dificultades de adquisición de insumos, equipamiento y personal capacitado, se suma la falta de registros adecuados de los formularios de investigación bacteriológica, que favorecería el incumplimiento de las técnicas requeridas para pesquisar tuberculosis en todas sus formas y mejorar el control del tratamiento. Es así que hay que mantener múltiples cometidos: una capacitación continua de los equipos locales que incentive la pesquisa a través del tamizaje de los sintomáticos respiratorios que consultan y el control mensual con baciloscopía durante el tratamiento de la $\mathrm{TB}^{12,13}$, maximizar el nivel operativo de la red de laboratorios para el control de la tuberculosis, manejar la TB multidrogorresistente (resistente a rifampicina e isoniazida: TBMDR $)^{2,4}$ e impulsar actividades de colaboración con otros programas ${ }^{14}$-de control de enfermedades respiratorias del adulto (ERA), Programa Cardiovascular que incluye a los diabéticos, intervenciones relacionadas con la co-infección (Programa VIH/SIDA) y realización de baciloscopías a embarazadas y pacientes postrados si se mantienen sintomáticos respiratorios.

Uno de los problemas es que actualmente se está usando una gran variedad de formularios de solicitud de investigación bacteriológica en el SSMS. Menos de la mitad contiene los enunciados adecuados a la norma (información VT/AT y drogas utilizadas en pacientes antes tratados y durante el control de tratamiento). De todas formas lo que especifica el 96,6\% (información VT/AT) ya permite decidir implementar las técnicas de cultivo y pruebas de susceptibilidad a las drogas en la red de laboratorios (toda vez que se registren los antecedentes expuestos en la Tabla 1). Una menor proporción (3,4\%), utiliza órdenes de otro tipo (de exámenes de bioquímica) que no provee ninguna referencia a tuberculosis. Se estima que el formulario de solicitud de baciloscopía no acompaña a la evolución de los procedimientos que se realizan actualmente en el laboratorio $\mathrm{y}$ se puede concebir que el tipo de formulario condicione la omisión y la calidad del llenado.

Esporádicamente otros establecimientos y servicios de salud han simplificado la solicitud de investigación bacteriológica, con formularios dobles auto-copiativos de pesquisa y de otro color para las BK de control de tratamiento e incluso se ha propuesto incluirla en el formulario de exámenes del perfil bioquímico, que sólo indica el nombre, el sistema numerado de identificación individual: "rol único nacional" (RUN) del paciente y la procedencia. Se pretende funcionalidad, minimizar las tareas y que sea más expedita, con el argumento de buscar después los antecedentes de las pocas muestran que resultan positivas. Sin embargo, es fácil imaginar el trabajo posterior de encontrar el domicilio, el consultorio que manejará al paciente (especialmente si no está inscrito al momento del diagnóstico) y los antecedentes para tomar decisiones en el laboratorio y en el programa de TB local de manera oportuna. Por otra parte, se plantea incluso a nivel nacional implementar la realización de BK con el agregado de cultivo de todas las muestras, y estudio de susceptibilidad a drogas antituberculosas de todos los cultivos que resulten positivos con colonias de micobacterias. Sin duda es un proyecto ambicioso, que supone aumento de los costos y del trabajo de los laboratorios de referencia y de la Sección Micobacterias del ISP. En la visita de la Organización Panamericana de la Salud (OPS) 
al Programa de Control de la Tuberculosis en Chile en 2013, se recomendó implementar un plan de fortalecimiento de la red de laboratorios, cumpliendo con los estándares de bioseguridad e incluso se contempla la descentralización de la técnica del cultivo del nivel intermedio ${ }^{11}$. Yáñez ha comentado que la inversión en el control de la TB tiene alta rentabilidad ${ }^{15}$.

Este estudio se basó en fuentes secundarias lo que aumenta el riesgo de sesgo, especialmente de información y se asume que los datos de la base que maneja el programa TB se completan y son veraces. En este estudio se buscó validez interna, que represente a la población y se pudo incluir todos los formularios con resultado $(+)$ del laboratorio de referencia, provenientes de la totalidad de las comunas del SSMS. A partir de 2013 en SSMS se intenta obtener un formulario único ideal, que incluya los criterios de la Norma del Programa y que contenga los factores de riesgo de TB y ya se iniciaron los trabajos de un formato electrónico con el sistema de información de la red asistencial (SIDRA) ${ }^{16}$. Una futura evaluación medirá si el sistema reduce los errores de registro y de confusión en el diagnóstico y seguimiento de los pacientes, mejorando la oportunidad y la efectividad de las acciones del laboratorio. Futuros estudios podrían generar un indicador de interés -el tiempo de demora entre la localización del caso (fecha de realización de la BK), el diagnóstico (fecha en que se obtuvo el resultado del laboratorio) y la instalación del tratamiento- que pudiera incentivar completar de manera fidedigna los registros, con implicancias para los pacientes y el logro de las metas.

\section{Conclusiones}

Un número importante de formularios de solicitud de investigación bacteriológica omite o registra erróneamente información clave para el proceder del laboratorio. La descripción de esta primera instancia de coordinación clínica-laboratorio hace recomendable un entrenamiento constante en este procedimiento en las capacitaciones $\mathrm{y}$ en las supervisiones del programa de TB.

Como actividad de salud pública el tamizaje con baciloscopía beneficia a toda la población y también involucra a todo el personal de salud ${ }^{2}$; es así que el formulario debe contener los factores de riesgo y los antecedentes de tratamiento antituberculoso claramente especificados, con la finalidad de lograr integrar un instrumento que mejore la oportunidad del diagnóstico y el buen seguimiento de los pacientes.

\section{Agradecimientos}

Los autores agradecen al personal de salud del Laboratorio de referencia del Complejo Asistencial Barros Luco (CABL) del SSMS por su apoyo en la obtención de los formularios de baciloscopías con el resultado de su trabajo, a la bioquímica Andrea Searle y a los médicos Pedro Zitko y Jorge Victoria por sus valiosos aportes.

\section{Bibliografía}

1.- FARGA V, CAMINERO J A. Tuberculosis. 3ra ed. Santiago, Chile: Editorial Mediterráneo; 2011.

2.- MINSAL. Manual de Organización y Normas Técnicas. Programa Nacional de Control de la Tuberculosis. Santiago, Chile: MINSAL; 2005.

3.- TOMAN K. Tuberculosis Detección de casos, tratamiento y vigilancia Preguntas y respuestas. $2^{\mathrm{a}}$ ed. Washington, D.C.: OPS, C); 2006.

4.- RODRÍGUEZ J C, IRAZOQUI P. Guías para el manejo de la tuberculosis resistente. OMS 2011. Rev Chil Enf Respir 2012; 28: 153-8.

5.- SCAPPATICCIO A, VELASCO M, LEIVA T, RODRÍGUEZ J C. Frecuencia de micobacterias ambientales en Chile en el año 2008. Rev Chil Enf Respir 2011; 27: 214-22.

6.- OMS. Definiciones y marco de trabajo para la notificación de tuberculosis- Revisión 2013. Ginebra: Organización Mundial de la Salud; [cited 201310 de octubre]; Disponible en: http://www.google.cl/url?sa=t\&rct=j\&q $=\&$ esrc $=$ s\&source $=$ web $\& \mathrm{~cd}=1 \&$ ved $=0 \mathrm{CCwQFjAA \& u}$ $\mathrm{rl}=\mathrm{http} \% 3 \mathrm{~A} \% 2 \mathrm{~F} \% 2 \mathrm{Fwww}$.paho.org $\% 2 \mathrm{Fhq} \% 2 \mathrm{Findex}$. php\%3Foption\%3Dcom_docman\%26task\%3Ddoc view\%26gid\%3D22984\%26Itemid\%3D\&ei=41JXUrK vCISfyQHrmID4Cg\&usg=AFQjCNHunRZXLbseVu Cwf6Cj-P_UEBjEuQ\&sig2=YzbEKaLCEBdscs42Bh MNMw\&bvm=bv.53899372,d.dmg

7.- MINSAL. Objetivos Sanitarios 2011-2020. Chile: MINSAL; 2011 [citado 201228 de junio]; Disponible en: http://www.slideshare.net/CesfamMTC/objetivossanitarios-decada-2011-2020.

8.- INE. Proyecciones de población. Chile: INE; 2012 [citado 20133 de octubre]; Disponible en: http://www. deis.cl/estadisticas-poblacion/

9.- STATA CORP LP. Stata Statistical Software. 11 ed. College Station TX2009.

10.- EL-SONY A, ENARSON D, KHAMIS A, BARAKA O, BJUNE G. Relation of grading of sputum smears with clinical features of tuberculosis patients in routine practice in Sudan. Int J Tuberc Lung Dis 2002; 6: 91-7.

11.- HERRERA T. La situación de la tuberculosis en Chile y los actuales desafíos. Visita de la OPS al programa de control de la tuberculosis de Chile. Rev Chil Enf Respir 2013; 29: 46-9.

12.- OKADA K, ONOZAKI I, YAMADA N, YOSHIYAMA 
T, MIURA T, SAINT S, et al. Epidemiological impact of mass tuberculosis screening: a 2-year follow-up after a national prevalence survey. Int J Tuberc Lung Dis 2012; 16: 1619-24.

13.- HARRIES A D, GAUSI F, SALANIPONI F M. When are follow-up sputum smears actually examined in patients treated for new smear-positive pulmonary tuberculosis. Int J Tuberc Lung Dis 2004; 8: 440-4.

14.- HERRERA T. VI Reunión de países de baja prevalencia de tuberculosis en Las Américas. Rev Chil Enf Respir 2013; 29: 108-12.

15.- YÁÑEZ A. Comentarios sobre los países de baja prevalencia de tuberculosis en América Latina. Rev Chil Enf Respir 2013; 29: 112-7.

16.- MINSAL. Sistema de Información de la Red Asistencial. Chile: MINSAL; 2010 [citado 201314 de octubre]; Disponible en: http://www.minsal.gob.cl/portal/url/ page/minsalcl/g_varios/sidra.html

Anexo

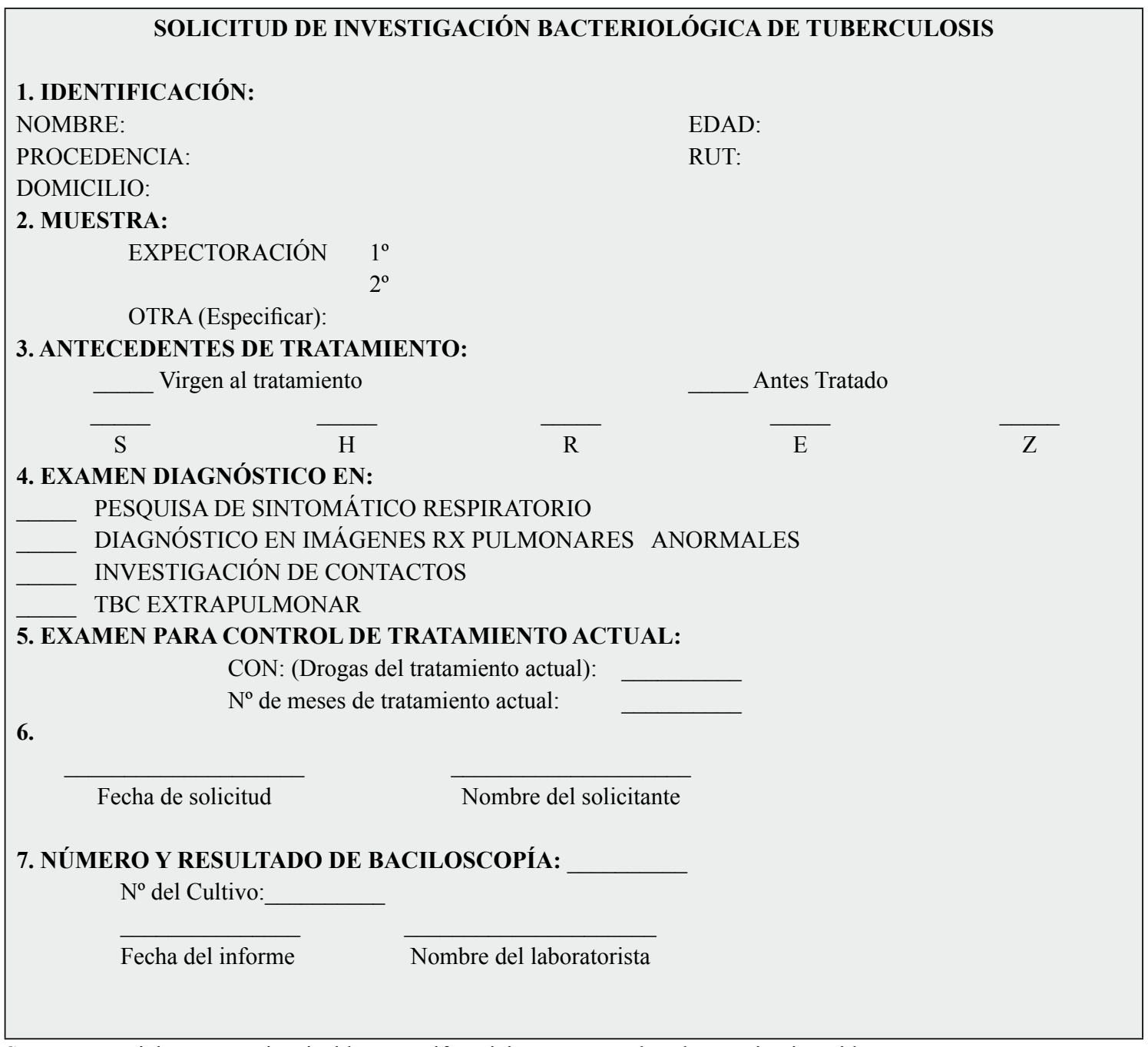

$\mathrm{S}=$ estreptomicina; $\mathrm{HIN}=$ isoniacida $; \mathrm{R}=$ Rifampicina; $\mathrm{E}=$ Etambutol; $\mathrm{Z}=$ Pirazinamida.

Correspondencia a:

Dra. Paulina Ramonda Celedón

Departamento Técnico en Salud del SSMS.

Santa Rosa 3453. San Miguel. Santiago, Chile.

E-mail: paulina.ramonda@redsalud.gov.cl 